\title{
Responsivity boosting in FIR TiN LEKIDs using phonon recycling: simulations and array design
}

Adalyn Fyhrie, Christopher McKenney, Jason Glenn, Henry G. LeDuc, Jiansong Gao, et al.

Adalyn Fyhrie, Christopher McKenney, Jason Glenn, Henry G. LeDuc, Jiansong Gao, Peter Day, Jonas Zmuidzinas, "Responsivity boosting in FIR TiN LEKIDs using phonon recycling: simulations and array design," Proc. SPIE 9914, Millimeter, Submillimeter, and Far-Infrared Detectors and Instrumentation for Astronomy VIII, 99142B (20 July 2016); doi: $10.1117 / 12.2231476$

Event: SPIE Astronomical Telescopes + Instrumentation, 2016, Edinburgh, United Kingdom 


\title{
Responsivity Boosting in FIR TiN LEKIDs Using Phonon Recycling: Simulations and Array Design
}

\author{
Adalyn Fyhrie ${ }^{\mathrm{a}}$, Christopher McKenney ${ }^{\mathrm{b}}$, Jason Glenn ${ }^{\mathrm{a}}$, Henry G. LeDuc ${ }^{\mathrm{c}}$, Jiansong Gao ${ }^{\mathrm{b}}$, \\ Peter Day ${ }^{\mathrm{c}}$, and Jonas Zmuidzinas ${ }^{\mathrm{d}}$ \\ ${ }^{a}$ University of Colorado at Boulder, $389 \mathrm{UCB}$, Boulder, CO, USA \\ bNational Institute of Standards and Technology, 325 Broadway, Boulder, CO, USA \\ ${ }^{\mathrm{c}}$ Jet Propulsion Laboratory, 4800 Oak Drive, Pasadena, CA, USA \\ ${ }^{\mathrm{d}}$ California Polytechnic Institute, 1200 E California Blvd, Pasadena, CA, USA
}

\begin{abstract}
To characterize further the cosmic star formation history at high redshifts, a large-area survey by a cryogenic $\sim 5$ meter class telescope with a focal plane populated by tens to hundreds of thousands of far-infrared (FIR, 30$300 \mu \mathrm{m}$ ) detectors with broadband detector noise equivalent powers (NEPs) on the order of $3 \times 10^{-19} \mathrm{~W} / \sqrt{\mathrm{Hz}}$ is needed. Ideal detectors for such a surveyor do not yet exist. As a demonstration of one technique for approaching the ultra-low NEPs required by this surveyor, we present the design of an array of $96350 \mu \mathrm{m}$ KIDs that utilize phonon recycling to boost responsivity. Our KID array is fabricated with TiN deposited on a silicon-on-insulator (SOI) wafer, which is a $2 \mu \mathrm{m}$ thick layer of silicon bonded to a thicker silicon wafer by a thin oxide layer. The thick silicon is etched away underneath the absorbers so that the inductors are suspended on just the $2 \mu \mathrm{m}$ membrane. The intent is that quasiparticle recombination phonons are trapped in the thin membrane, thereby increasing their likelihood of being re-absorbed by the KID to break additional Cooper pairs and boost responsivity. We also present a Monte-Carlo simulation that predicts the amount of signal boost expected from phonon recycling given flexible detector geometries and illumination strategies. For our initial array geometry, the simulation predicts a small but likely measurable $50 \%$ boost in responsivity.
\end{abstract}

Keywords: kinetic inductance detector, phonon, far-infrared, simulation

\section{INTRODUCTION}

The far-infrared (FIR, 30-300 $\mu \mathrm{m}$ ) is a critical band for characterizing the cosmic star formation history at high redshifts. To achieve a more complete understanding of this history, a large-area survey by a cryogenic $\sim 5$ meter class telescope with a focal plane populated by tens to hundreds of thousands of FIR detectors is needed. With a primary aperture cooled to 4 Kelvin and equipped with sufficiently sensitive detectors, the sensitivity of such a surveyor would only be limited by astrophysical backgrounds and source confusion. Broadband detector noise equivalent powers (NEPs) on the order of $3 \times 10^{-19} \mathrm{~W} / \sqrt{\mathrm{Hz}}$ are required to be photon noise limited. ${ }^{1}$

Kinetic inductance detectors (KIDs) show great promise for a mission such as this because of their intrinsic frequency multiplexability and straightforward cryogenic amplification requirements. De Visser et al. ${ }^{2}$ demonstrated NEPs in the low $10^{-19} \mathrm{~W} / \sqrt{\mathrm{Hz}}$ range with antenna-coupled low-volume aluminum KIDs, meeting the sensitivity requirements in the range needed for the aforementioned surveyor. However, the power dissipation of the readout electronics is driven by the relatively high readout frequencies (4-8 GHz) employed.

We fabricated a 96 lumped element KID (LEKID) array using TiN whose high resistivity results in low readout frequencies around $200 \mathrm{MHz}$, thereby decreasing the cost and weight of readout electronics, an important consideration for space missions. TiN's high resistivity also necessitates a high filling fraction to obtain a good match between the effective surface impedance of the TiN absorber and the wave impedance in silicon, thereby achieving high optical efficiency. However, the high area fill fraction also corresponds to a large detector volume, which reduces responsivity. To compensate, we introduce the concept of phonon recycling, an effort to boost

Send correspondence to A. Fyhrie: adfy9371@colorado.edu

Millimeter, Submillimeter, and Far-Infrared Detectors and Instrumentation for Astronomy VIII, edited by Wayne S. Holland, Jonas Zmuidzinas, Proc. of SPIE Vol. 9914, 99142B

(C) 2016 SPIE · CCC code: 0277-786X/16/\$18 · doi: 10.1117/12.2231476

Proc. of SPIE Vol. 9914 99142B-1 

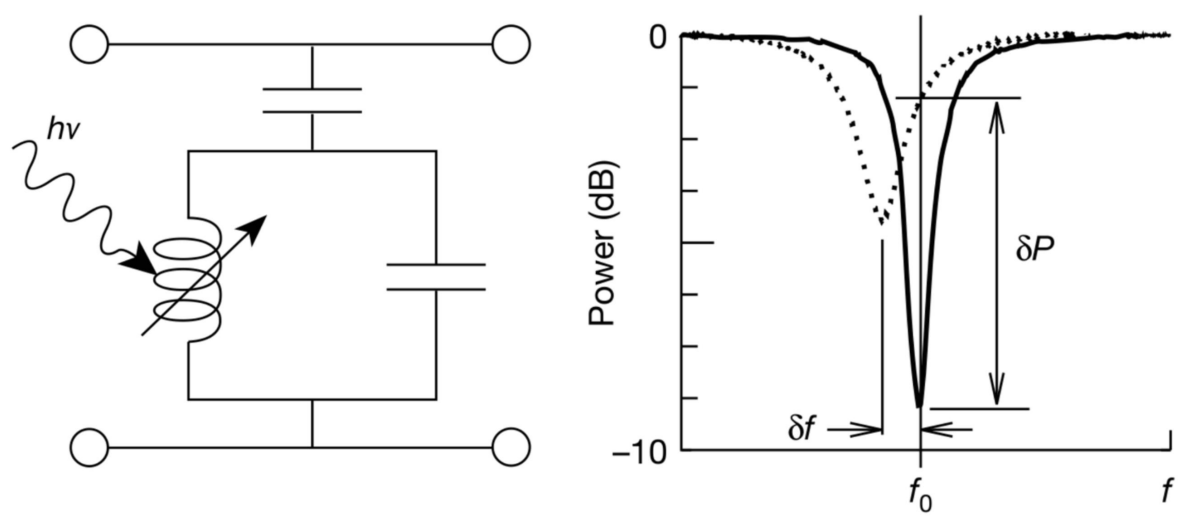

Figure 1. From Day et al. ${ }^{3}$ Left: an equivalent circuit representation of a KID, the inductor shown as variable due to absorbed radiation. The upper capacitor is the coupling capacitor to the readout line. Right: the power transmitted through the circuit measured in $\mathrm{dB}$ as a function of frequency. The KID absorbs its resonance frequency $f_{0}$, creating a dip. The solid line is the KID response before absorbing photons, and the dotted line is after absorbing photons.

responsivity and reduce NEP, and explain this idea in depth in in Section 2.2. In short, the mechanism of "phonon recycling" refers to trapping the phonons created by photo-produced quasiparticles recombining into Cooper pairs. These recombination phonons, if trapped in the vicinity of the detector's active area, can be reabsorbed in the superconductor, breaking more Cooper pairs and thereby enhancing the kinetic inductance response.

In this paper, we first define the concept of phonon recycling and describe how it is achieved by backthinning silicon below the KID active detector area. Secondly, we describe the array that we fabricated in the Microdevices Laboratory at the Jet Propulsion Laboratory to test the concept of phonon recycling. We then present our Monte-Carlo simulations that predict the amount of signal boost expected from phonon recycling given flexible detector geometries and illumination schemes. Finally, we present our plans for investigating the effects of phonon recycling in our array, which will simultaneously test the validity and completeness of our simulation and indicate the utility of phonon recycling for boosting responsivity.

\section{TECHNICAL BACKGROUND}

\subsection{Kinetic Inductance Detectors}

Kinetic inductance detectors are superconducting $L C$ circuits with resonant frequencies $f_{0}=1 /(2 \pi \sqrt{L C})$. An equivalent circuit diagram and the resulting resonant dip (or absorption feature) from this $L C$ circuit are shown in Figure 1. The capacitance $C$ is set by the design of the interdigitated electrode structure. The inductance $L$ has two components: first, the geometric inductance which is resultant from the layout of the circuit and the metal from which it is made. Second, the kinetic inductance $\left(L_{k i}\right)$ which is provided by the superconducting charge carriers called Cooper pairs. The ratio of the kinetic to the total inductance, $\alpha=L_{k i} /\left(L_{k i}+L_{\text {geom }}\right)$, is called the kinetic inductance fraction. For our TiN KIDs, the kinetic inductance dominates and $\alpha \approx 1$.

Cooper pairs are electrons of opposite spin bound by an energy $2 \Delta_{0}$, which is related to the critical temperature $T_{C}$ of the superconductor: $\Delta_{0}=1.76 k_{B} T_{C}$. When a photon with energy $h \nu>2 \Delta_{0}$ is absorbed by the detector, the photon can break a Cooper pair into its individual electrons called "quasiparticles". Broken Cooper pairs have two effects on the readout of the system, illustrated in the right panel of Figure 1:

1. The kinetic inductance of the circuit is inversely proportional to the number of Cooper pairs in the system. Therefore, when Cooper pairs are broken the kinetic inductance of the circuit increases, thereby decreasing the resonant frequency $f_{0}$.

2. Quasiparticles increase the resistance (dissipation of energy) of the circuit, which in turn decreases the quality factor $Q$ of the circuit. $Q$ is defined by $Q=f_{0} / \Delta f$, where $f_{0}$ is the resonant frequency of the circuit and $\Delta f$ is the frequency difference between the $-3 \mathrm{~dB}$ points in the absorption feature. 


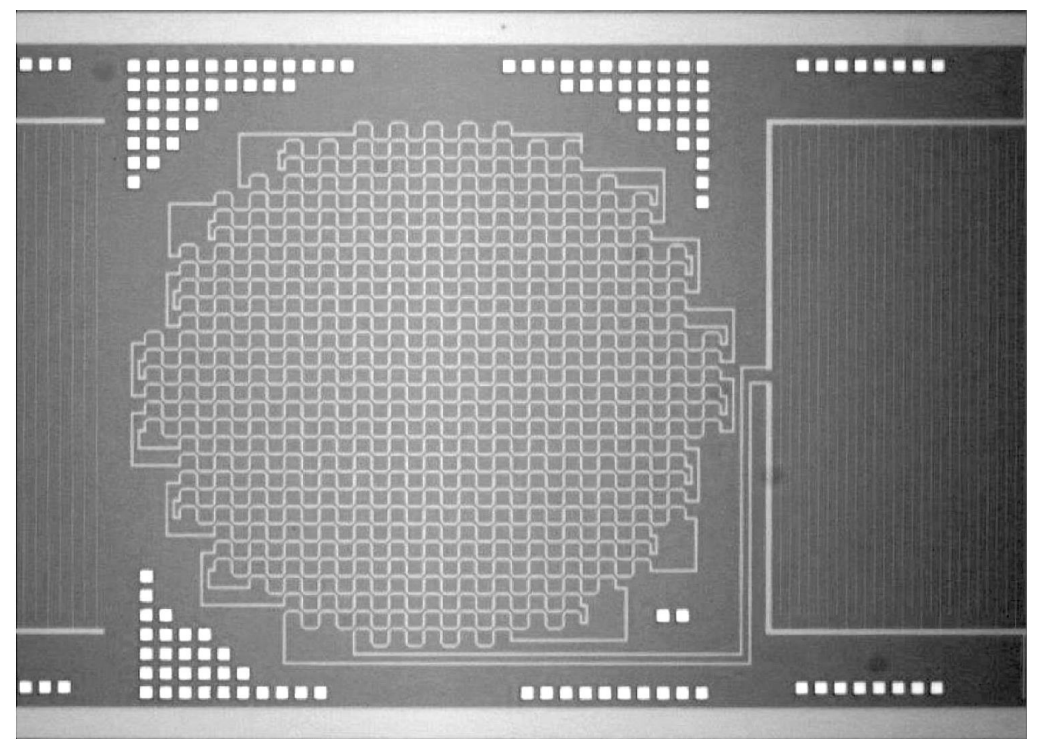

Figure 2. A picture of a single non-thinned detector with the same inductor and capacitor layout as the back-thinned devices. The roughly circular TiN meander is the inductor which also acts as the optical absorber, and is fully visible. For scale, the diameter of the inductor is $0.4 \mathrm{~mm}$. The rectangular interdigitated capacitor, also made of TiN, is only partially visible on the right hand side of the image. In a phonon recycled device, the silicon in the circular area below the inductor would be thinned to allow trapping of phonons below the inductor, which is the detector's active area.

\subsection{Phonon Recycling}

In order to achieve lower NEP detectors one can either decrease the noise or increase the responsivity of the detectors to light, because

$$
\mathrm{NEP}=\frac{\sqrt{S}}{R_{x}}
$$

where $S$ is the power spectrum of fractional frequency fluctuations and the responsivity $R_{x}$ is defined as the fractional change in resonance frequency for a given change in the incident optical power. The technique of phonon recycling focuses upon increasing $R_{x}$ by elongating the duration of the kinetic inductance response.

The process of phonon recycling begins when Cooper pairs, previously broken by absorbed radiation, recombine after an average time of $\tau_{q p} \approx 100 \mu \mathrm{s}{ }^{4-6}$ the quasiparticle recombination time. When Cooper pairs recombine, they release energy as a recombination phonon with $E=2 \Delta_{0}$. This recombination phonon usually escapes into the substrate upon which the superconductor is deposited. In phonon recycled devices, we design the array such that this recombination phonon is instead trapped underneath the detector's active area, increasing its probability of re-absorption and consequently breaking another Cooper pair. Cooper pairs broken by the first recombination phonon will again eventually recombine, releasing another recombination phonon which could then go on to break another Cooper pair.

The number of times this quasiparticle recombination and phonon re-absorption process repeats is $R-1$, where $R$ is called the "recycling factor". Emission and re-absorption of the phonon increases the effective quasiparticle recombination time from $\tau_{q p, 0}$ to

$$
\tau_{q p, r e c}=R \times \tau_{q p, 0} .
$$

This elongation of the signal increases the responsivity $R_{x}$ proportionally, since $R_{x} \propto \tau_{q p} \cdot{ }^{4}$

\section{ARRAY GEOMETRY}

We fabricated a 96 pixel array of $20 \mathrm{~nm}$ thick TiN deposited on a silicon-on-insulator (SOI) wafer. Each pixel consists of a meandered inductor/radiation absorber with a roughly circular outline and an interdigitated 

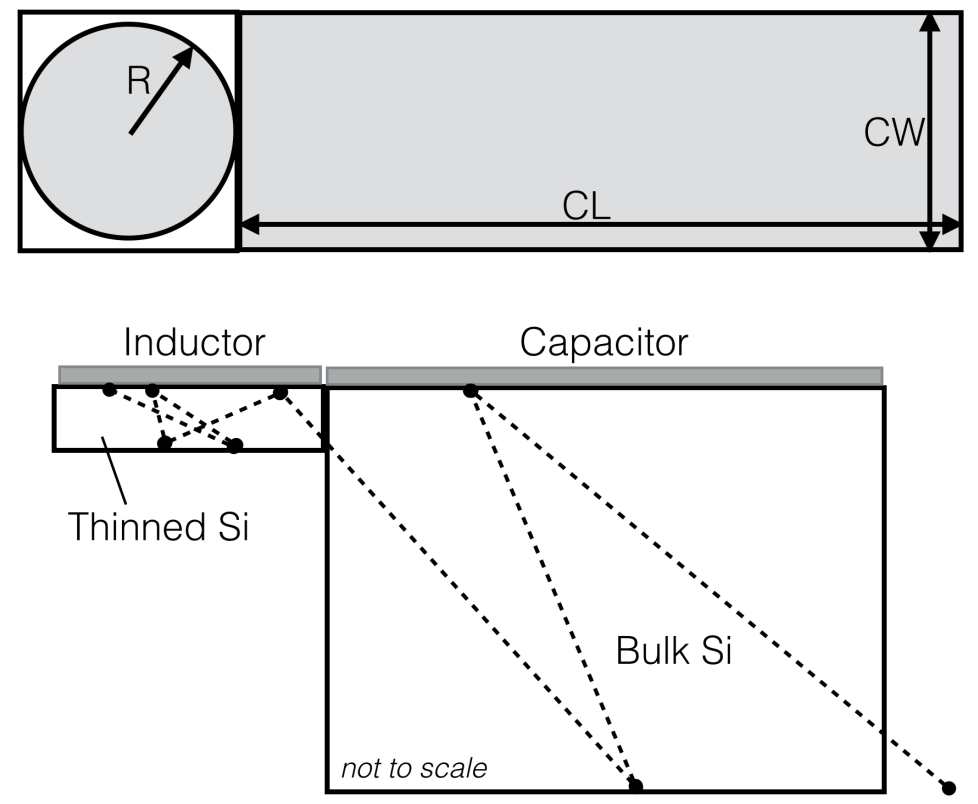

Figure 3. Geometry of the phonon recycling simulation. Top: A top-down view of the rectangular detector cell, where the solid black lines define boundaries between distinct regions and the grey shaded areas are where superconducting metal has been deposited. The rectangular outline on the right is the interdigitated capacitor with dimensions $C W$ by $C L$. The circular outline with radius $R$ is the inductor/absorber. The white area surrounding the inductor is bare silicon. Bottom: A side view of a lengthwise cut of the detector cell, showing the thick bulk silicon below the capacitor area and thinned silicon below the inductor. Again, grey shaded areas are the superconducting metal deposition areas. An example of a possible phonon path is shown as a dashed line, where the phonon begins in the inductor, travels into the capacitor area, and ends beyond the detector cell. Each scattering point off of a surface is represented as a solid black circle.

capacitor to mitigate two-level-system noise. ${ }^{7}$ A picture of a single detector is shown in Figure 2. The readout frequencies were designed to be a few hundred $\mathrm{MHz}$ with quality factors on the order of $3 \times 10^{4}$. The array is meant to be coupled to radiation with a lenslet array, which will focus radiation onto the inductor.

To trap the phonons below the detector active area (the inductor) and achieve phonon recycling, the SOI is etched away in a circular pattern below the inductor so that the inductors are suspended on the $2 \mu \mathrm{m} \mathrm{Si}$ membrane. In our array, half of the detectors have thinned Si below their inductors and half of the detectors remain atop the full thickness of the SOI wafer. Rows of thinned/non-thinned detectors alternate on the array.

\section{SIMULATIONS OF PHONON RECYCLED DEVICES}

Using Python, we created a Monte-Carlo style simulation of phonons scattering within a single detector to predict what boost in responsivity would result from the detector geometry described in Section 3. The simulation is generalizable to many different detector geometries and illumination strategies, making it a useful tool for exploring the utility of phonon recycling in KID devices.

\subsection{Simulation Geometry and Phonon Behavior}

The simulation uses a three-dimensional geometry shown in Figure 3. A rectangular capacitor with a filling fraction $\eta_{f i l l, C}$, width $C W$, and length $C L$ sits atop a silicon block of thickness $t$. To its right, a circular inductor with a filling fraction $\eta_{f i l l, L}$ and radius $R$ sits atop a silicon block of thickness $t_{\text {thin }}<t$. The entire detector cell is a rectangle of length $C L+2 R$ and width $C W$, with any area not covered by the inductor or capacitor consisting of bare silicon. The thickness of the TiN that comprises the inductor and the capacitor is $t_{T i N}$.

Phonons are initiated in random positions at the top of the detector cell, as if they had been created by Cooper pairs recombining within the TiN of the detector. Different illuminations of the detector are represented 

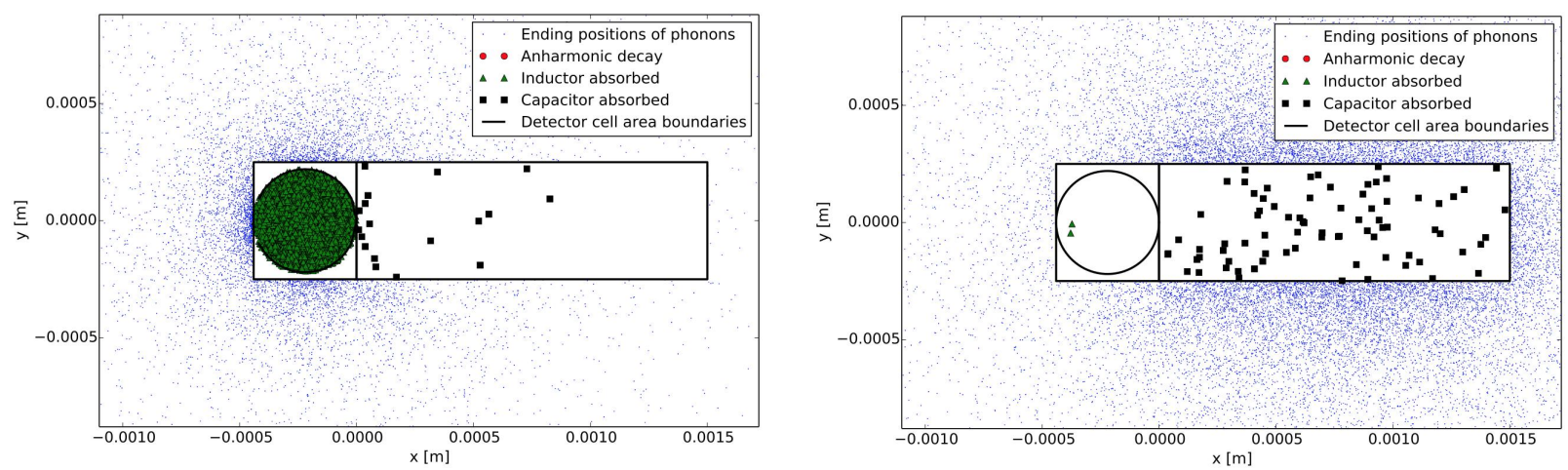

Figure 4. The ending locations of escaped phonons (blue dots) and locations of re-absorption (and consequent re-emission) of phonons, given that they start at a random location within the inductor (Left) or within the capacitor (Right). In this run of 30,000 phonons, the thinned silicon is $2 \mu \mathrm{m}$ thick and the bulk silicon below the capacitor is $350 \mu \mathrm{m}$ thick. Absorptions in the inductor are shown in green triangles, while absorptions in the capacitor are shown in black squares. Absorptions in the inductor are much more common than in the capacitor due to the higher number of bounces the phonon experiences within the thinned silicon below the inductor, giving a higher number of opportunities for re-absorption. In the bare Si area around the inductor the phonons cannot be absorbed, but the phonon is allowed to continue propagating as it is still considered within the boundary of the rectangular detector cell. If the phonon's total distance travelled exceeds the anharmonic decay length of phonons in silicon $\left(>1 \mathrm{~m}^{8}\right)$, then its path is terminated and it is labelled as anharmonically decayed (red circles). Instances of anharmonic decay are observed to occur only at steep scattering angles when the phonon travels a great distance in one bounce; therefore, they do not show up on this plot which zooms in to view the area close to the detector. In addition, the anharmonic decay length is larger than the array itself, and therefore is included for completeness but can generally be neglected.

by the phonon start positions: for uniform illumination the phonons start within both the capacitor and the inductor, while for illumination focused on the detector active area the phonons start only within the inductor. To separate the impact on the recycling factor of phonons beginning in the capacitor from those beginning in the inductor, we simulate phonons starting within the capacitor only and the inductor only.

After initiation, phonons can then scatter at any angle and travel in a straight trajectory to the bottom of the detector cell. From the bottom, they again scatter at any angle and travel back to the top of the detector cell where the TiN is deposited. This isotropic scattering of phonons off of silicon surfaces is based upon the measurements reported by D. Marx and W. Eisenmenger in $1982 .{ }^{9}$ Phonons travel in this manner until they leave the outer rectangular boundary of the detector cell.

Any time the phonon hits the top of the detector cell, it has a probability of re-absorption $\eta_{r}$ by the TiN, on the order of 0.01 , given by

$$
\eta_{r}=T_{\text {acoustic }} \eta_{f i l l} 2 t_{T i N} / l_{p b}
$$

where $T_{\text {acoustic }}$ is the acoustic matching factor of order unity, ${ }^{10} \eta_{\text {fill }}$ is the filling fraction of the metal in the area where the phonon hits (inductor or capacitor), $t_{T i N}$ is the thickness of the TiN, and $l_{p b}$ is the mean free path to pair breaking in the superconductor. $l_{p b}=v_{p h o n o n} \tau_{p b}$ is not well measured for TiN, however, Kaplan et al. $1976^{11}$ report that the phonon lifetime against pairbreaking $\tau_{p b}$ is $\propto T_{c}$. Therefore, $\mathrm{Al}$ and TiN's comparable $T_{c}$ 's should have similar pair breaking lengths (to first order), and we use Al's value of $l_{p b} \approx 1 \mu \mathrm{m}$ as an approximation for $l_{p b}$ in these simulations. Evaluation of $l_{p b}$ using the known properties of $\mathrm{TiN}^{11-14}$ gives a value for $\eta_{r}$ that is larger by a factor of a few, which we take to be reasonable agreement. Equation 3 is an approximation as well, and assumes that the phonon travels straight up and down in the TiN with a resultant total path length in the TiN of $2 t_{T i N}$. Averaging over the angle of incidence would increase the effective path length and introduce an extra numerical factor, but this level of refinement is premature at this stage.

After reabsorption, the phonon is then re-emitted in a random direction and can continue to bounce between the top and bottom of the silicon at random angles with opportunities for re-absorption and emission until it leaves the boundary of the detector cell. The most important output of this simulation is the number of times a single phonon is reabsorbed by the TiN in the inductor $\left(N_{a b s}\right)$, which yields the recycling factor $R=N_{a b s}+1$ 


\begin{tabular}{|c|c|}
\hline Thinned Si Thickness $[\mu \mathrm{m}]$ & Recycling Factor $\mathrm{R}$ \\
\hline 2 & 1.49 \\
\hline 0.5 & 3.0 \\
\hline 0.25 & 4.9 \\
\hline 0.1 & 10.5 \\
\hline
\end{tabular}

Table 1. The simulated recycling factor $R$ for a given thinned Si thickness, assuming all phonons start within the inductor. The geometry for these simulations is: inductor radius of $220 \mu \mathrm{m}$, inductor filling fraction of $1 / 3$, capacitor lengths of $1500 \mu \mathrm{m}$, capacitor widths of $550 \mu \mathrm{m}$, capacitor filling fraction of 0.25 , and TiN thicknesses of $20 \mathrm{~nm}$. These parameters match the geometry of the back-thinned detectors analyzed in this paper.

as described in Section 2.2. The ending positions as well as locations of re-absorptions are shown in Figure 4 for two different phonon starting positions: randomly within the inductor only or randomly within the capacitor only.

\subsection{Simulation Results and Predictions}

One major result of the simulation is that the recycling factor $\mathrm{R}$ does not depend on the phonon start position, which is equivalent to detector illumination. The right panel of Figure 4 shows that for 30,000 phonons starting within the capacitor, only two instances of phonon re-absorption in the inductor occur. We postulate that this extremely small number of inductor re-absorptions is due to the small solid angle seen by phonons when passing from the thick silicon into the thinned silicon below the inductor, effectively preventing phonons in the capacitor from entering the area below the inductor. Therefore, only phonons initiated in the inductor contribute to the phonon recycling signal, meaning that $\mathrm{R}$ is largely illumination independent.

Another major result of these simulations is the dependency of the recycling factor $R$ on the thickness of $\mathrm{Si}$ below the inductor: as the membrane of $\mathrm{Si}$ below the inductor gets thinner, $R$ increases dramatically. Table 1 shows the recycling factor $R$ for a given thickness of Si below the inductor given a simulation geometry that matches the array of phonon-recycled KIDs analyzed in this paper. For a single detector with the same geometry as those detectors described in Section 3, a recycling factor of $R=1.49$ is simulated.

To further increase $R$ one could increase the radial size of the inductor, prolonging the amount of time the phonon spends bouncing below the inductor before it escapes from the detector cell and providing more opportunities for reabsorption. One could also increase the thickness of the TiN or the filling fraction of the inductor because this increases the phonon re-absorption probability according to Equation 3. However, we are limited in this approach by the necessity of impedance matching the superconductor to the silicon substrate. Additionally, the increased volume of superconductor decreases the responsivity ${ }^{4}$ due to the lower quasiparticle density. These problems are avoided by focusing on phonon trapping by thinning the silicon substrate, as we have in our array design.

\section{DISCUSSION AND FUTURE WORK}

To determine the functionality of back-thinning silicon to achieve phonon recycling and measure the recycling factor $R$ (Equation 2) for our array, we must measure $\tau_{q p}$ for all detectors and compare the non-thinned $\tau_{q p, 0}$ with the thinned silicon detectors' $\tau_{q p, r e c}$. Rows of thinned and un-thinned devices alternate on the array, meaning that functional phonon recycling would result in two alternate rows with elevated $\tau_{q p}$.

To measure $\tau_{q p}$, we will flash the array with a white LED at $100 \mathrm{~Hz}$ while driving a single resonator at its resonance frequency $f_{0}$ and record the exponential response of $d f / f_{0}$ to the flashing LED. The exponential rolloff timescale $\tau_{\text {exp }}$ is a measure of the longest timescale of the system, either $\tau_{q p}$ or the resonator ringtime $\tau_{\text {ring }}$ which can be measured by fitting each detector's $S_{21}$. Phonon recycling is meant to elongate $\tau_{q p}$, likely making it the longest timescale of the system and measurable by this pulsing LED technique.

Ultimately, the KID array will be coupled to incoming radiation with a lenslet array. A silicon back-plate with cylinders in the back-thinned inductor locations will ensure that there is less than a $\lambda / 4$ gap of air between the back-illuminated devices and the lenslet. 
The simulation presented above is a tool we will use to optimize the array geometry to achieve maximal phonon recycling. As an example of the parameter space we have to work with, Table 1 demonstrates how thinner membrane layers can improve the recycling factor by many times. Careful consideration must be given to the tradeoffs between improved phonon recycling geometries and their possible detrimental effects upon KID noise or responsivity, as discussed in Section 4.2. As part of this analysis, we will expand the simulation to include multiple detector cells and evaluate the importance of phonon cross-talk between detectors.

\section{ACKNOWLEDGEMENTS}

This work was funded by the Research and Technology Development Fund of the Jet Propulsion Laboratory.

\section{REFERENCES}

[1] L. Armus, e. a., "From early galaxies to habitable planets: The science case and concept for a far-infrared surveyor." White Paper (2015).

[2] de Visser, P., Baselmans, J., Bueno, J., and Klapwijk, T., "Demonstration of an NEP of $3.8 \times 10^{-19}$ $\mathrm{W} / \mathrm{Hz}^{1 / 2}$ at $1.54 \mathrm{THz}$ in multiplexible superconducting microresonator detectors," in [2014 39th International Conference on Infrared, Millimeter, and Terahertz waves (IRMMW-THz)], 1-2 (2014).

[3] Day, P., LeDuc, H. G., Mazin, B., Vayonakis, A., and Zmuidzinas, J. Nature 425, 817-821 (2003).

[4] Zmuidzinas, J., "Superconducting microresonators: Physics and applications.," Annual Review of Condensed Matter Physics 3(1), 169-214 (2012).

[5] Henry G. Leduc, e. a., "Titanium nitride films for ultrasensitive microresonator detectors," Applied Physics Letters $97(10), 2509$ (2010).

[6] Jiansong Gao, e. a., "A titanium-nitride near-infrared kinetic inductance photon-counting detector and its anomalous electrodynamics," Applied Physics Letters 101.14, 142602 (2012).

[7] Gao, J., The physics of superconducting microwave resonators, PhD thesis, California Institute of Technology. (2008).

[8] Maris, H. J. and Tamura, S.-I., "Anharmonic decay and the propagation of phonons in an isotopically pure crystal at low temperatures: Application to dark-matter detection," Physical Review B 47, 727-739 (1993).

[9] Marx, D. and Eisenmenger, W., "Phonon scattering at siliconcrystal surfaces," Zeitschrift für Physik B Condensed Matter 48(4), 277-291 (1982).

[10] Kaplan, S. B., "Acoustic matching of superconducting films to substrates," Journal of Low Temperature Physics 37.3-4, 343-365 (1979).

[11] Kaplan, S. B., Chi, C. C., Langenberg, D. N., Chang, J. J., Jafarey, S., and Scalapino, D. J., "Quasiparticle and phonon lifetimes in superconductors," Phys. Rev. B 14, 4854-4873 (1976).

[12] Sanjeev K. Gupta, e. a., "First-principles studies of the superconductivity and vibrational properties of transition-metal nitrides TMN (TM= Ti, V, and Cr).," Materials Chemistry and Physics 143.2, 503-513 (2014).

[13] Eyvaz I. Isaev, e. a., "Phonon related properties of transition metals, their carbides, and nitrides: A firstprinciples study," Journal of applied physics 101.12, 123519 (2007).

[14] Z. Dridi, e. a., "First-principles calculations of vacancy effects on structural and electronic properties of TiCx and TiNx," Journal of Physics: Condensed Matter 14.43, 10237 (2002). 